\title{
Comparison of chromosomal and array-based comparative genomic hybridization for the detection of genomic imbalances in primary prostate carcinomas
}

\author{
Franclim R Ribeiro, 1,2, Rui Henrique 3,4, Merete Hektoen², Marianne Berg2, \\ Carmen Jerónimo ${ }^{1,4,5}$, Manuel R Teixeira*1,4 and Ragnhild A Lothe ${ }^{2,6}$
}

Address: ${ }^{1}$ Department of Genetics, Portuguese Oncology Institute - Porto, Porto, Portugal, ${ }^{2}$ Department of Cancer Prevention, Institute for Cancer Research, The Norwegian Radium Hospital, Oslo, Norway, ${ }^{3}$ Department of Pathology, Portuguese Oncology Institute - Porto, Porto, Portugal, ${ }^{4}$ Department of Pathology and Molecular Immunology, Institute of Biomedical Sciences, University of Porto, Porto, Portugal, ${ }^{5}$ Fernando Pessoa University, Porto, Portugal and ${ }^{6}$ Department of Molecular Biosciences, University of Oslo, Oslo, Norway

Email: Franclim R Ribeiro - frsr@netcabo.pt; Rui Henrique - rmhenrique@icbas.up.pt; Merete Hektoen - merete.hektoen@labmed.uio.no; Marianne Berg - marianne.berg@labmed.uio.no; Carmen Jerónimo - cjeroni@ufp.pt; Manuel R Teixeira* - mteixeir@ipoporto.min-saude.pt; Ragnhild A Lothe - rlothe@radium.uio.no

* Corresponding author

Published: 04 September 2006

Molecular Cancer 2006, 5:33 doi:10.1 186/1476-4598-5-33
Received: 15 August 2006

Accepted: 04 September 2006

This article is available from: http://www.molecular-cancer.com/content/5/I/33

(C) 2006 Ribeiro et al; licensee BioMed Central Ltd.

This is an Open Access article distributed under the terms of the Creative Commons Attribution License (http://creativecommons.org/licenses/by/2.0), which permits unrestricted use, distribution, and reproduction in any medium, provided the original work is properly cited.

\begin{abstract}
Background: In order to gain new insights into the molecular mechanisms involved in prostate cancer, we performed array-based comparative genomic hybridization (aCGH) on a series of 46 primary prostate carcinomas using a I Mbp whole-genome coverage platform. As chromosomal comparative genomic hybridization ( $\mathrm{cCGH}$ ) data was available for these samples, we compared the sensitivity and overall concordance of the two methodologies, and used the combined information to infer the best of three different aCGH scoring approaches.

Results: Our data demonstrate that the reliability of aCGH in the analysis of primary prostate carcinomas depends to some extent on the scoring approach used, with the breakpoint estimation method being the most sensitive and reliable. The pattern of copy number changes detected by aCGH was concordant with that of $\mathrm{CCGH}$, but the higher resolution technique detected 2.7 times more aberrations and $\mathbf{I} 5.2 \%$ more carcinomas with genomic imbalances. We additionally show that several aberrations were consistently overlooked using $c \mathrm{CGH}$, such as small deletions at $5 q, 6 q$, $12 p$, and $17 p$. The latter were validated by fluorescence in situ hybridization targeting TP53, although only one carcinoma harbored a point mutation in this gene. Strikingly, homozygous deletions at I0q23.3I, encompassing the PTEN locus, were seen in $58 \%$ of the cases with $10 q$ loss.

Conclusion: We conclude that aCGH can significantly improve the detection of genomic aberrations in cancer cells as compared to previously established whole-genome methodologies, although contamination with normal cells may influence the sensitivity and specificity of some scoring approaches. Our work delineated recurrent copy number changes and revealed novel amplified loci and frequent homozygous deletions in primary prostate carcinomas, which may guide future work aimed at identifying the relevant target genes. In particular, biallelic loss seems to be a frequent mechanism of inactivation of the PTEN gene in prostate carcinogenesis.
\end{abstract}




\section{Background}

Prostate cancer is a frequent and heterogeneous malignancy with few established prognostic markers. Increased knowledge on the genetic basis of this condition is expected to significantly improve the clinical management of these patients. Most of the genetic data currently available on this malignancy has been obtained using chromosomal comparative genomic hybridization (cCGH), a whole-genome screening methodology well established in the scientific field [1]. We have recently published a statistical dissection of the cCGH data available in the literature and proposed two main genetic pathways involved in prostate carcinogenesis, starting either with $8 \mathrm{p}$ or $13 \mathrm{q}$ deletions [2]. We showed that $8 \mathrm{q}$ gain and $13 q$ loss were good predictors of progression into locally invasive disease and that losses of $6 \mathrm{q}$ and $10 \mathrm{q}$ were significantly associated with metastatic cancers. In addition, some of these genetic changes have shown prognostic value independently of tumor grade and stage [3-6].

The recent advent of microarray-based platforms for the detection of genome-wide copy number changes promises to uncover novel recurrent genetic aberrations and provide a more accurate delineation of genomic regions previously known to be altered in different cancer types. However, there is still no consensus regarding the scoring of array-based comparative genomic hybridization (aCGH) results, making it difficult to objectively compare findings obtained by different platforms and analysis tools. A few aCGH studies of prostate cancer cell lines have been reported [7-11], but most cell lines grow as stable, uncontaminated cell populations with clonal karyotypes. This makes the comparison of different platforms and scoring methods easier than for clinical samples, which often contain varying degrees of non-neoplastic cell contamination and thus fail to show the fluorochrome ratio intensities expected for low-level copy number changes. Whole-genome aCGH findings have been reported in small subsets of primary prostate carcinomas [12-14], and high-resolution platforms have been developed to study recurrently affected genomic regions $[14,15]$. However, Paris et al. were the first to use the aCGH methodology to study a larger series of clinical prostate cancer samples $[16,17]$. The particular scoring methodology used in those studies resulted in the detection of a large percentage of single clone alterations of unclear significance. Furthermore, the concordance between the previously established chromosomal CGH and the new array-based CGH platforms could not be conclusively evaluated, since genetic information obtained with the former method was available only for a small subset of the samples.

In the present study, we systematically compared aCGH and cCGH profiles of 46 primary prostate carcinomas and determined the best aCGH scoring methodology to delineate genomic copy number changes relevant for prostate carcinogenesis.

\section{Results Quality control}

Clones that failed to produce a result in more than $60 \%$ of the sample set were removed from further analysis, as were those displaying copy number changes in at least two negative controls. Clones with known polymorphic regions were not present in the array. Additionally, analysis of the dye-swap experiments and negative controls suggested a dye-specific affinity of several clones on chromosome $\mathrm{X}$ and $\mathrm{Y}$, which are rich in repetitive sequences. As these seemed to produce copy number aberrations (not previously detected by cCGH) in all samples, we chose to remove them from the analysis. From the 3568 clones in the microarray, 2787 passed these stringent quality criteria. The median percentage of clones remaining per sample (out of 2787) was $97 \%$ in the negative controls, $96 \%$ in the biopsy samples and $89 \%$ in the prostatectomy series.

\section{Comparison of scoring methods}

Sample-specific fixed-thresholds, even when stringently determined, provided a fragmented genetic profile in which several low-level copy number changes (CNCs) were not scored and a large number of single clone aberrations (average of 5.8 per sample) as well as false positive findings (average of 3.7 CNCs per control) were obtained. The data segmentation approach provided by CGH-Plotter was also affected by low intensity ratios, resulting in most gains and several deletions, confirmed to be present using cCGH, being missed. On the other hand, the number of single clone aberrations ( 0.5 per sample), as well as false positive findings (0.1 CNCs per control), was greatly reduced. aCGH-Smooth, by focusing on the detection of contiguous groups of clones with similar mean intensities, was able to score a large number of gains and losses with intensities that did not reach theoretical ratios for a stroma-free tumor sample. This strategy thus detected twice as much CNCs than the previous ones, with the advantage of producing very few single-clone aberrations (average of 1.4 per sample) and virtually no false positive findings (average of $0.1 \mathrm{CNCs}$ per control). Due to their uncertain significance, the few single clone aberrations were not included in the final scoring. Figure 1 provides a schematic representation of the individual profiles produced by the three aCGH scoring methodologies tested on a sample with known copy number aberrations.

\section{Comparison between cCGH and aCGH findings}

aCGH confirmed $95 \%$ of the 146 copy number changes detected by cCGH in the 46 prostate carcinomas (Figure 
A

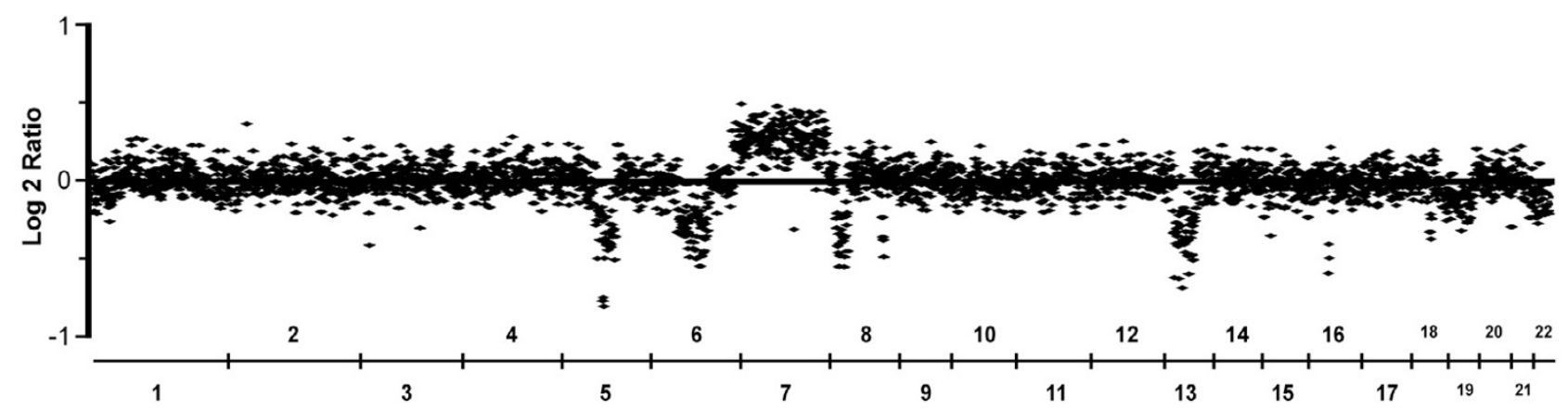

B

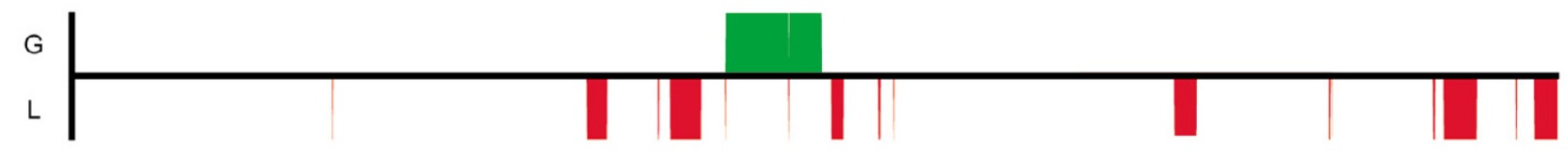

C

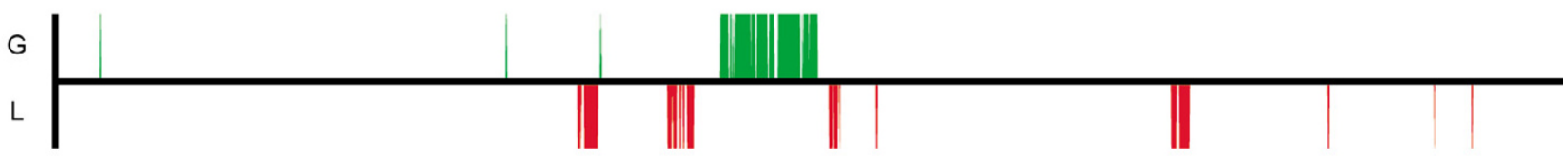

D

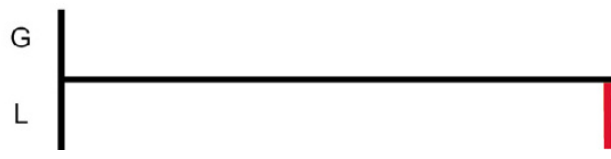

Figure I

Comparison of aCGH score results for sample "Bp22" using different automated scoring approaches. (A) Normalized log-2 ratios, with clones ordered according to their genomic position. Note that the theoretical intensity values for gains and losses are not reached. (B) Results using aCGH-Smooth. (C) Results using sample-specific fixed thresholds calculated in Normalization Suite. (D) Results using CGH-Plotter. For the purposes of visualization and comparison, all diagrams were generated in Microsoft Excel based on data provided by the different analysis tools, and thus do not correspond to the visual outputs provided by each individual software.

2). Most of the non-confirmed aberrations involved single chromosomal bands located at chromosomal ends. Seven cases without copy number changes by cCGH were found to have genomic aberrations upon aCGH analysis, representing a $15.2 \%$ detection increase of abnormal cases. Regarding individual aberrations, aCGH detected 2.7 times more copy number changes than cCGH (347 versus $146)$. Forty-five percent of the gained regions spanned more than 50 clones, whereas a large proportion of lost regions involved 20 to 50 clones (33.8\%). Overall, 73.2\% of all gains and $70 \%$ of all losses involved at least 10 clones, which corresponds roughly to the $10 \mathrm{Mb}$ resolution level estimated for CCGH. Up to $60 \%$ of the gains and $50 \%$ of the losses larger than 10 clones had been detected using cCGH. Specifically, deletions of $5 q(p=0.066), 6 q$ $(p=0.065), 12 p(p=0.014)$, and 17p $(p=0.072)$ were particularly overlooked by cCGH, whereas deletions at $8 \mathrm{p}$ and gains of 7 and $8 \mathrm{q}$ were detected by both techniques in almost identical proportions.

\section{aCGH profile of prostate carcinomas}

Overall, 38 cases (83\%) displayed copy number changes, with losses of genomic material being 2.7 times more frequent than gains (Figure 2, Table 1). Seven out of 15 cases without copy number changes by cCGH were found to harbor aberrations upon aCGH analysis. Interestingly, 7/ 24 prostatectomy and 1/22 biopsy samples did not display copy number changes even at this level of resolution. Regions of recurrent genomic loss were located at $8 \mathrm{p}$ (67\%), 5q (39\%), 16q (37\%), 6q (35\%), 13q (33\%), 10q (33\%), 17p (30\%), 12p (24\%), and 2q (20\%), whereas frequent copy number gains were observed at $8 \mathrm{q}(30 \%)$, $7(22 \%)$, and $3 q(13 \%)$ [see Additional file 1]. Amplifications were detected in a total of four biopsy samples (Table 2), whereas homozygous deletions were detected in a total of 10 carcinomas (Figure 3, Table 2), most frequently at 10q23.31 (seven out of 12 tumors with 10q loss showed homozygous deletion at this locus, involving a minimum common region of 4 clones). 
A

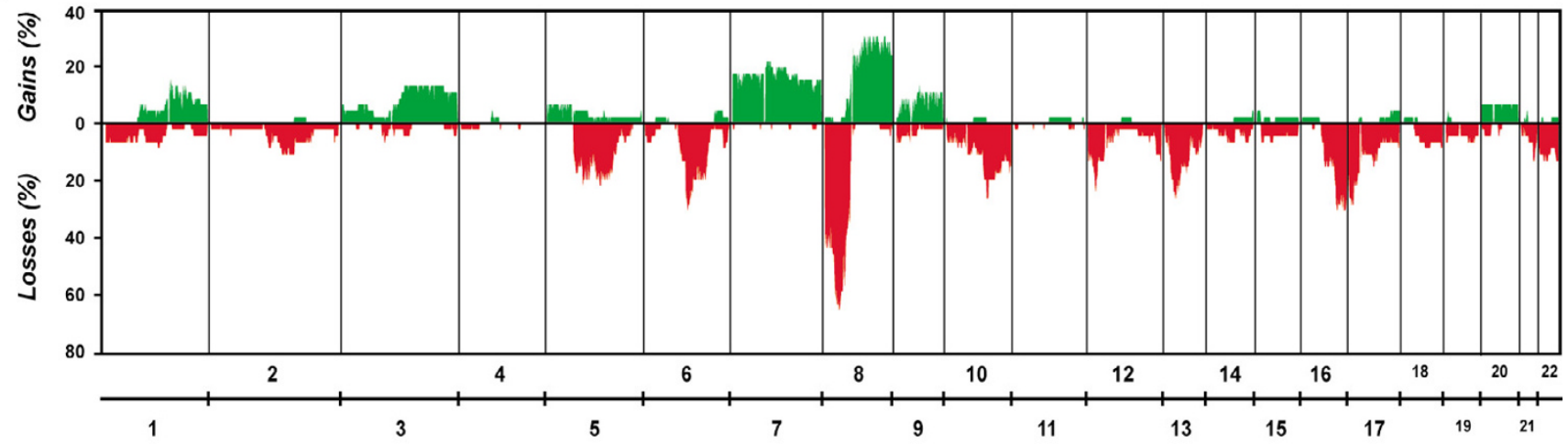

$\mathrm{B}$

Clones (n=2787) ordered along chromosomes 1 to 22

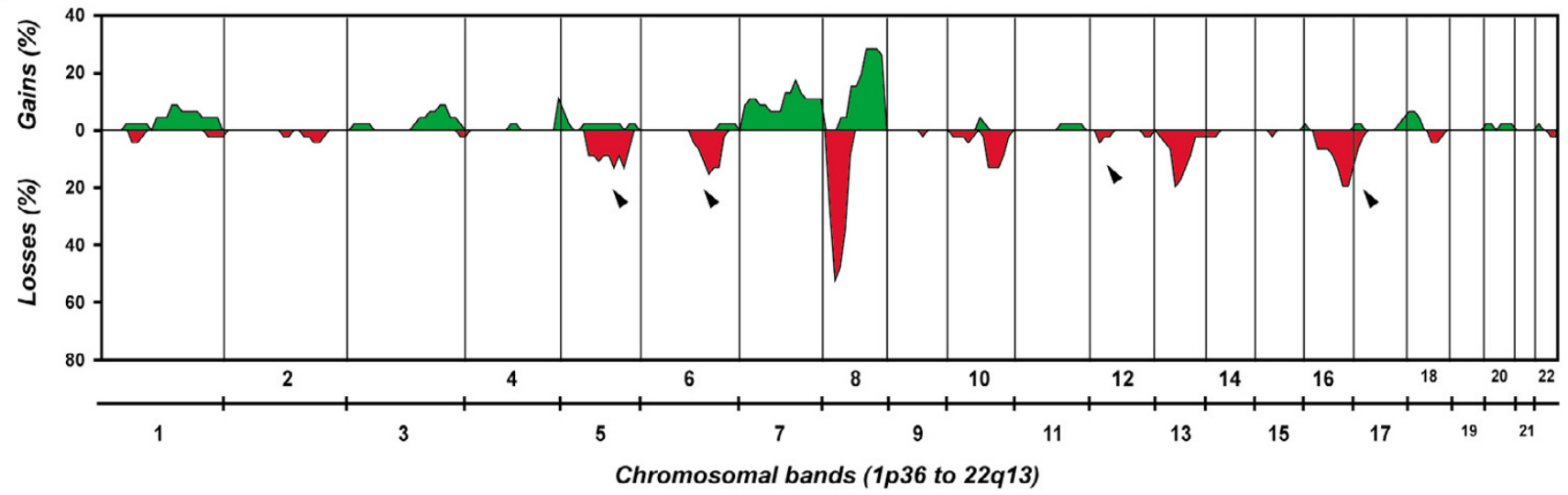

Figure 2

Genomic findings in 46 primary prostate carcinomas. (A) Array-CGH findings (38 samples with copy number changes). (B) Chromosomal CGH findings (3I samples with copy number changes). Arrows indicate relevant differences in the frequency of aberrations detected by both techniques.

\section{FISH and mutation analyses of TP53}

Hybridization was successful in all 10 paraffin embedded core biopsies analyzed by dual-color FISH. These corresponded to samples with $(\mathrm{n}=7)$ and without $(\mathrm{n}=3)$ deletions at $17 \mathrm{p} 13$ detected by aCGH. FISH results confirmed the loss of one or more copies of the TP53 probe (compared to the control probe) in all but one of the cases with $17 \mathrm{p}$ loss (Figure 4; the exception was a case deemed uninformative due to the small size of the paraffin section analyzed). The remaining three samples displayed a normal fluorescent pattern with two signals for both the centromeric and the 17p probes. Regarding TP53 mutation screening of the 51 samples, including the nine with $17 \mathrm{p}$ losses by cCGH, aCGH, or FISH, only one mutation (exon 5, codon 177, CCC->CTC, Pro-> Leu) and a known polymorphism (exon 6, codon 213, CGA->CGG, Arg->Arg, detected in four samples) were present, all in cases without a 17 p13 deletion.

\section{Discussion}

In this work we used array-based CGH to assess the genomic profile of a large series of primary prostate carcinomas. As these samples had previously been analyzed using chromosomal CGH, we were able to compare the two techniques in terms of sensitivity and overall performance, and to test distinct automated scoring approaches for aCGH data. Whereas most scoring methods will achieve concordant results if a given sample is pure and the hybridization quality is excellent, clinical samples usually contain non-neoplastic cell populations that influence the interpretation of the results. In the particular case of the prostate gland, the enriched cellular content of the stromal component should not be underestimated. Combined with the variability within chromosome spreads (in cCGH), labeling efficiency, and hybridization behavior, a certain level of methodological noise/variability is expected that may seriously influence 
Table I: Overview of aCGH findings in $\mathbf{4 6}$ prostate cancer samples

\begin{tabular}{|c|c|c|c|c|}
\hline & Frequency $(n=46)$ & SRO' & Size $(\mathrm{Mb})$ & Genomic position (Mb) \\
\hline \multicolumn{5}{|c|}{ Losses } \\
\hline $8 p$ & $67.4 \%$ & $8 p 21.2-8 p 22$ & 12.0 & $15.36-27.36$ \\
\hline $5 q$ & $39.1 \%$ & $\begin{array}{c}5 q \mid 3.1 \\
5 q|3.2-5 q| 3.3 \\
5 q 21.1 \\
5 q 22.2-5 q 23.1\end{array}$ & $\begin{array}{l}0.6 \\
2.1 \\
2.0 \\
7.7\end{array}$ & $\begin{array}{c}66.79-67.41 \\
71.74-73.87 \\
99.03-101.09 \\
112.07-119.75\end{array}$ \\
\hline $16 q$ & $37.0 \%$ & $\begin{array}{l}16 q 12.1-16 q \mid 2.2 \\
16 q 22.2-16 q 22.3 \\
16 q 23.3-16 q 24.1\end{array}$ & $\begin{array}{l}4.1 \\
1.7 \\
3.6\end{array}$ & $\begin{array}{l}49.70-53.86 \\
70.70-72.37 \\
81.20-84.83\end{array}$ \\
\hline $6 q$ & $34.8 \%$ & $\begin{array}{l}6 q|4 .|-6 q \mid 4.3 \\
6 q|6.2-6 q 22.3|\end{array}$ & $\begin{array}{c}3.5 \\
23.6\end{array}$ & $\begin{array}{c}82.59-86.12 \\
99.38-123.02\end{array}$ \\
\hline $10 q$ & $32.6 \%$ & $\begin{array}{l}10 \mathrm{q} 23.31 \\
10 \mathrm{q} 26.3\end{array}$ & $\begin{array}{l}1.0 \\
2.6\end{array}$ & $\begin{array}{c}89.69-90.69 \\
|30.0|-\mid 32.63\end{array}$ \\
\hline $13 q$ & $32.6 \%$ & $\begin{array}{c}|3 q| 4 .|-| 3 q \mid 4.3 \\
|3 q 2| .32-|3 q 2| .33\end{array}$ & $\begin{array}{l}5.5 \\
4.7\end{array}$ & $\begin{array}{l}39.49-45.03 \\
66.42-71.16\end{array}$ \\
\hline $17 p$ & $30.4 \%$ & $|7 p| 3.1-|7 p| 3.2$ & 2.3 & $6.14-8.49$ \\
\hline $12 p$ & $23.9 \%$ & $12 p \mid 3.2$ & 1.9 & $10.93-12.86$ \\
\hline \multicolumn{5}{|c|}{ Gains } \\
\hline $8 q$ & $30.4 \%$ & $\begin{array}{l}8 q|1.2|-8 q \mid 2.1 \\
8 q \mid 3.2-8 q 24.23\end{array}$ & $\begin{array}{l}10.8 \\
68.2\end{array}$ & $\begin{array}{c}48.76-59.73 \\
68.36-136.56\end{array}$ \\
\hline 7q & $21.7 \%$ & $\begin{array}{l}7 q 1|.21-7 q 1| .22 \\
7 q 21.1 \mid-7 q 22.1\end{array}$ & $\begin{array}{c}6.3 \\
19.5\end{array}$ & $\begin{array}{l}64.63-71.01 \\
80.26-99.76\end{array}$ \\
\hline
\end{tabular}

Aberrations occurring in less than $20 \%$ of the samples are not displayed.

I Smallest region of overlap (often more than one per chromosomal arm).

the analysis. In a recent paper by Lai et al. [18], several automated scoring methodologies were compared using datasets recreating distinct aberrations and background noise, and only a few were able to reliably score low-level copy number changes. Taking this information into account, and using our cCGH data as a starting point, we compared three freely available analysis tools representing common aCGH scoring methodologies. We found fixed-thresholds to be extremely affected by the quality of the hybridization and the presence of normal cells, which resulted in known alterations being missed completely or scored only partially. The large number of single-clone aberrations obtained also rendered the distinction between true copy number changes and false positive results subject to interpretation and additional validation. The data segmentation approach of CGH-Plotter produced minimal levels of single clone aberrations, but was unable to detect most low-intensity changes. Finally, aCGH-Smooth consistently detected low-level copy number changes with only residual levels of single-clone aberrations and false positive findings, thus providing a more sensitive and reliable approach to the scoring of our $1 \mathrm{Mb}$ BAC array data.

Using this analytical tool, 95\% of the changes detected using cCGH were confirmed by aCGH. The theoretical 10fold increase in resolution of aCGH resulted in an increase of $15.2 \%$ in the proportion of genetically abnormal prostate carcinomas and in the detection of 2.7 times more copy number aberrations. Strikingly, of the aberrations involving more than $10 \mathrm{Mb}$ (the estimated resolution limit of cCGH), 45\% had not been scored using cCGH. We believe this discrepancy reflects two limitations of cCGH, namely the lack of sensitivity in detecting lowintensity alterations (independently of the size of the aberration) and the inherent difficulty in scoring regions of metaphase chromosomes of smaller size and variable hybridization behavior (17p, 18p, 19, 20, 21, and 22). It is noteworthy that deletions at $8 \mathrm{p}$ and gains at $8 \mathrm{q}$ and 7 were equally detected by both methodologies, whereas deletions at 5q, 6q, 12p, and 17p were particularly overlooked using cCGH. Paris et al. [17] have previously reported such a comparison in a series of 20 formalinfixed paraffin embedded prostate cancers. In their work, $90 \%$ of the cCGH copy number changes were confirmed by aCGH, which detected 3.4 times more alterations. As they used fixed thresholds to score their data, however, $44 \%$ of the aCGH findings consisted of single clone aberrations, thus requiring careful interpretation and validation.

The overall profile obtained for our prostate cancer samples was comparable to that described in previous aCGH studies of clinical samples $[13,16,17]$. It is noteworthy that most gains were detected in the overall more advanced group of carcinomas sampled by biopsy, whereas $77 \%$ of the alterations in the prostatectomy series corresponded to deletions, which according to the literature are the most common events in prostate carcinogenesis [2]. Interestingly, seven prostate cancers sampled by 
A

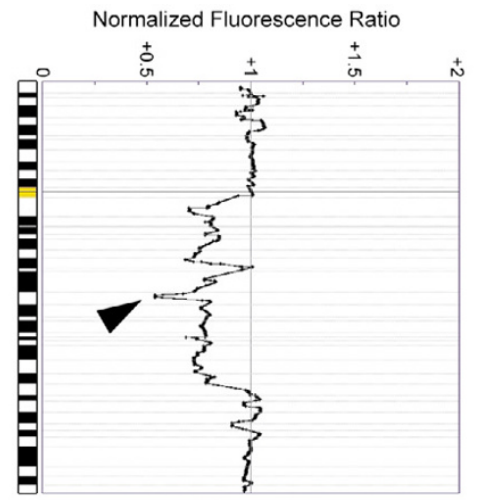

Chr \#5

B

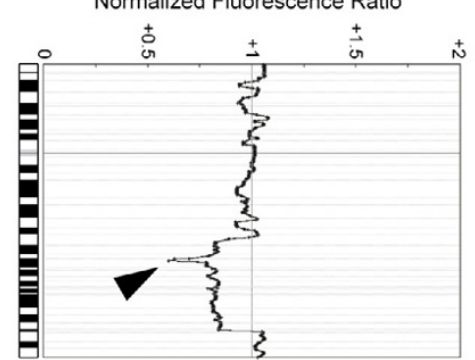

Chr \#10

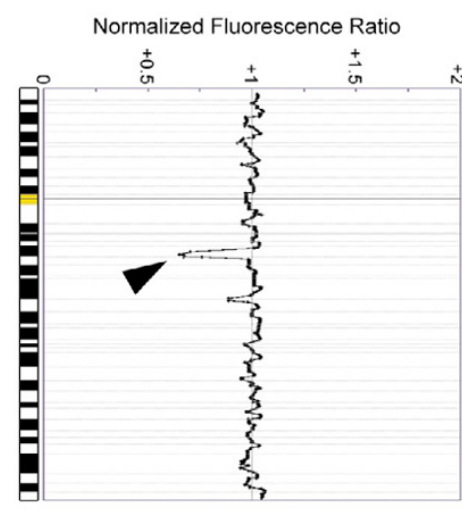

Chr \#5

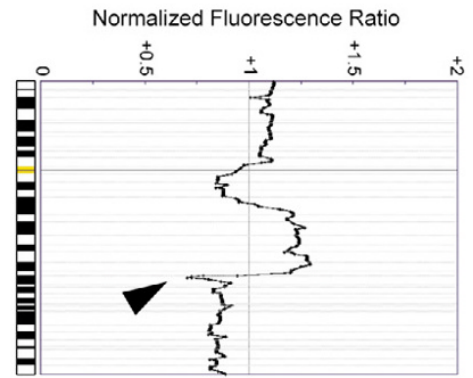

Chr \#10

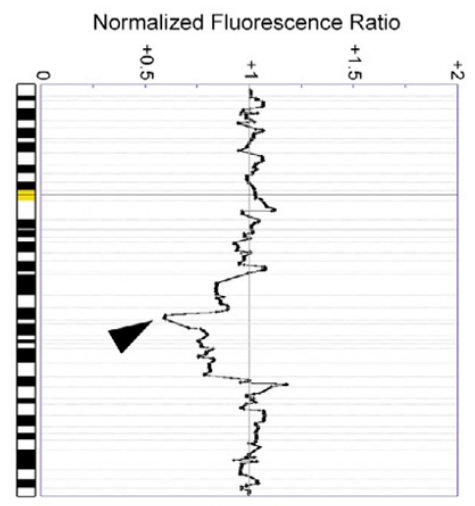

Chr \#5

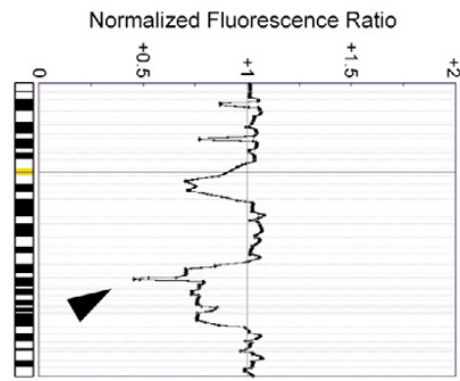

Chr \#10

Figure 3

Examples of homozygous deletions revealed by aCGH (arrow heads). (A) Homozygous deletions at different regions of 5q. (B) Recurrent homozygous deletions at 10q23.3I, encompassing the PTEN gene region.

prostatectomy (early staged tumors) did not display copy number changes even at this level of resolution, whereas losses at $8 \mathrm{p}$ and $16 \mathrm{q}$ and gain at $8 \mathrm{q}$ were already present in a considerable percentage of clinically confined carcinomas, indicating these alterations arose early during tumor progression. We and others have in fact shown that

Table 2: Amplifications and homozygous deletions detected in $\mathbf{4 6}$ prostate cancer samples

\section{Cytoband $\left(n^{\circ}\right.$ cases $)$ \\ Size (Mb) \\ Genomic position (Mb)}

Homozygous deletions

Amplifications

1.10

10

$\begin{array}{cc}5 q \mid 3 . I(I) & 0.62 \\ 5 q \mid 5(I) & 0.97 \\ 5 q 21.1-5 q 2 \mid .2(I) & 2.01 \\ 10 q 23.3 \mid(7) & 1.10 \\ |I q 23.2-1| q 23.3(I) & 1.10 \\ & \\ 6 q 24.1-6 q 25.3(I) & 13.80 \\ 7 q|1.22-7 q| I .23(I) & 5.90 \\ 7 q 22.1(I) & 0.72 \\ 8 p \mid 2(I) & 1.96 \\ 8 q 22.2-8 q 22.3(I) & 3.17 \\ 8 q 23.2-8 q 24.22(I) & 23.83 \\ |I q 22.3-1| q 23.1(I) & 3.8 I \\ 17 p \mid I .2(I) & 0.99 \\ \mid 7 q 23.2-17 q 23.3(I) & 1.40 \\ 19 p \mid 3.3(I) & 0.98\end{array}$

$66.79-67.41$
$93.62-94.59$
$101.08-103.09$
$89.60-90.70$
$\mid 13.80-114.90$

$\mid 42.29-156.09$
$68.97-74.87$
$98.59-99.31$
$36.43-38.39$
$\mid 01.35-104.52$
$\mid 11.82-135.65$
$107.37-111.18$
$\mid 9.18-20.17$
$56.75-58.15$
$5.63-6.61$

$66.79-67.4$

3.80

5.90

1.96

3.17

3.81

0.99

0.98 
A

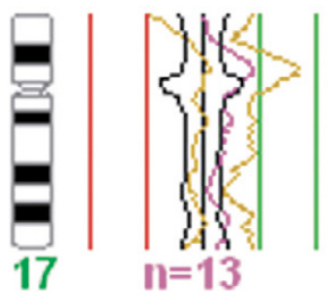

B
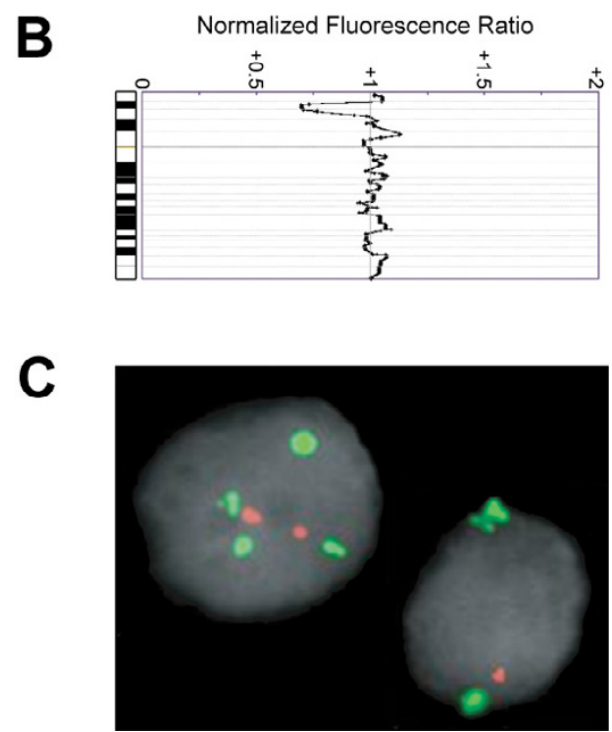

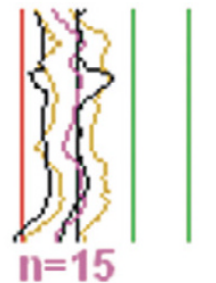

Normalized Fluorescence Ratio
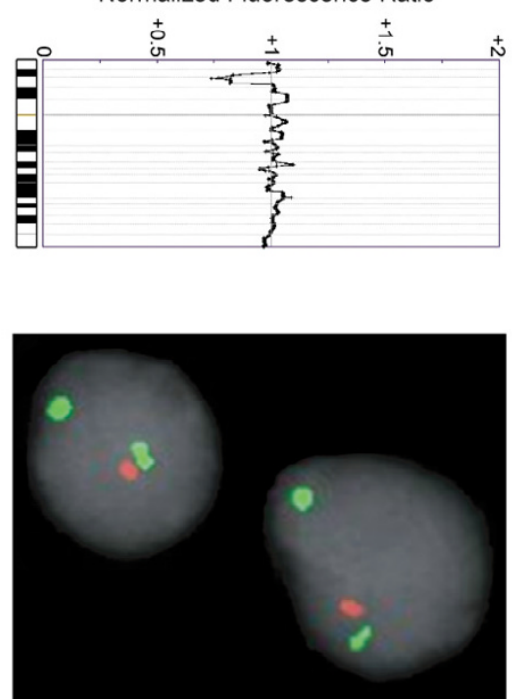
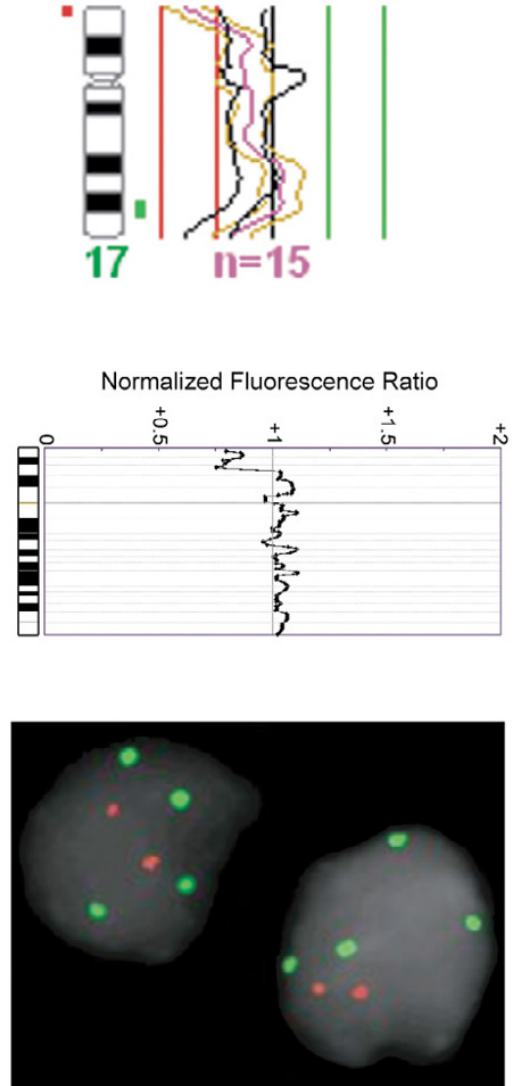

Figure 4

Genomic findings using cCGH, aCGH, and FISH in three selected biopsy samples. (A) cCGH results for chromosome 17, with a terminal $17 \mathrm{p} / 3$ deletion detected in only one case. (B) aCGH findings showing loss of $17 \mathrm{p} / 3$ in all three cases. (C) FISH findings confirming the loss of one or more TP53 copies (red) compared to the centromeric probe for chromosome 17 (green) in all three cases.

$8 \mathrm{q}$ gain is significantly associated with increased tumor grade and worse patient outcome $[3,5,6,19]$, therefore suggesting that some early cancer foci already carry genetic features of bad prognosis, whereas others do not display copy number changes at all and possibly correspond to a subset of less aggressive or even latent lesions.

Loss of $17 \mathrm{p}$, another alteration previously associated with poor prognosis and frequently overlooked by cCGH, often occurred together with $8 \mathrm{q}$ gain in our sample set. This led us to perform FISH and mutation screening for the most likely candidate at this location, the TP53 gene. Mutation frequencies for TP53 are extremely variable in prostate cancer studies (ranging from 3-45\%), but overall it is consensual that most clinically confined tumors have no mutations, whereas metastatic and androgen independent cancers harbor a high frequency of TP53 mutations $[20,21]$. We detected only one mutation in our set of clinically confined carcinomas, which is in accordance with the literature and suggests that this genetic event is more important for the progression, rather than to the establishment, of prostatic carcinomas. Loss of $8 p$, on the other hand, is an early and frequent finding in prostate cancer with no significant differences between cCGH and aCGH. It involves a minimum region of overlap spanning $\sim 12 \mathrm{Mb}$ (8p21.2 to 8p22) that encompasses over 50 confirmed genes with distinct cellular functions, making it difficult to pinpoint single candidate targets. Our and previous aCGH studies have been unable to find homozygous deletions at this chromosome arm, suggesting that many genes in this region may thus be working together on a dosage dependent manner to induce the initial stages of prostate carcinogenesis [22,23].

Deletions at chromosomal region $10 \mathrm{q}$ are also a frequent finding in prostate cancer cells, albeit associated with advanced disease. In 12 out of 15 cases with $10 \mathrm{q}$ loss in our series, a common region at 10q23.31 ( 1 Mbp long) was affected. Strikingly, in seven of these 12 carcinomas the deletion was homozygous. The only cancer-relevant 
gene from the few candidates at this location is PTEN/ MMAC1 [24,25], as it has already been shown that PTEN expression is reduced in a large subset of advanced prostate cancers $[26,27]$. Recent work on mouse models [2830] suggests that the absence of functional PTEN confers proliferating cells the ability to overlook apoptosis even when subjected to apoptotic stimuli. Haploinsufficiency of PTEN seems to already have a dramatic influence on the cellular response to apoptosis [28], with the loss of the second allele being actively selected for during disease progression [30]. Interestingly, analyses of this multifunctional protein phosphatase generally describe very low mutations frequencies [31-34], which further indicates that homozygous deletions, rather than mutations or epigenetic silencing, are the major mechanism of gene inactivation at this locus. This hypothesis has been recently strengthened by the recurrent finding of homozygous deletions encompassing the PTEN region in several prostate cancer cell lines and xenografts [35,36], as well as in primary tumors [37]. Homozygous deletions affecting 5q were also relatively frequent in our series of primary prostate carcinomas, but these were heterogeneous and the potential target genes remain unknown.

\section{Conclusion}

We conclude that aCGH can significantly improve the detection of genomic aberrations in cancer cells as compared to previously established whole-genome methodologies, although stromal contamination may significantly influence the sensitivity and specificity of most automated scoring approaches. The increased resolution of aCGH revealed several previously undetected aberrations and refined the breakpoints of those already found by cCGH. The recurrent regions of copy number gains and losses in primary prostate carcinomas highlighted in this study, as well as the novel amplified loci and frequent homozygous deletions, may guide future work aimed at identifying the relevant target genes.

\section{Materials and methods \\ Prostate carcinoma samples}

We have previously reported the genomic findings detected by cCGH in a series of prostatectomy specimens containing cancer [2] and in a series of fine- needle biopsies from prostate cancer suspects [6]. For the present aCGH study, 24 samples from the former and 22 samples from the latter series were selected, because we wanted to include early-staged tumors, as well as samples from more advanced, genetically complex cancers. From the prostatectomy series, in which all samples contained $>70 \%$ tumor cells, cases were selected to equally represent different Gleason score categories. From the biopsy series, only samples with morphological evidence of tumor were used and selection was based mostly on DNA availability. From the selected samples, $6 / 22$ biopsies and 9/24 pros- tatectomies had displayed no copy number changes using cCGH. The same DNA stocks were used for cCGH and aCGH. Additionally, a total of 51 carcinomas for which good quality DNA was available (46 samples from Ribeiro et al., 2006a, including the 24 selected for this aCGH study, and 5 biopsy samples from Ribeiro et al., 2006b, also included in the present report) were evaluated for TP53 gene mutations. Several paraffin-embedded tissue blocks corresponding to biopsy samples analyzed by aCGH were also selected for FISH validation studies.

\section{Array-based comparative genomic hybridization} Clone set

We used the Human $4 \mathrm{k}$ Genome-wide $1 \mathrm{Mb}$ resolution Arrays provided by the Norwegian Microarray Consortium (National technology platform supported by the functional genomics program of the Research Council of Norway [38]). Each slide consists of 3568 BAC/PAC probes positioned along the genome at an average resolution of $1 \mathrm{Mb}$, printed in duplicate onto two identical blocks in the array, for a total of four replicates per clone. Probe DNA was obtained from the $1 \mathrm{Mb} \mathrm{BAC} / \mathrm{PAC}$ clone set kindly provided by Dr. Nigel Carter at the Wellcome Trust Sanger Institute, UK [39], amplified using DOPPCR, and spotted onto CodeLink slides (Amersham Biosciences, Chalfont St Giles, UK) using a MicroGrid II arrayer (BioRobotics, Boston, USA). Mapping information (clone location and cytogenetic bands) was retrieved from the Ensembl Human Genome Browser v36, December 2005 freeze [40].

\section{Labeling and hybridization}

DNA from the 46 prostate samples had been extracted using standard methods. The same commercially available male control DNA (Promega Corporation, Madison, WI) was used as reference for all samples. For each experiment, $500 \mathrm{ng}$ of test and reference DNA were digested with Dpn II (New England Biolabs, Ipswich, MA), purified using the QIAquick PCR purification kit (Qiagen Inc, Valencia, CA), and labeled with Cy3-dCTP (test) or Cy5dCTP (reference) (PerkinElmer, Boston, MA) in a random-primer reaction with the BioPrime Array CGH Genomic Labeling Kit (Invitrogen, Paisley, UK). Unincorporated nucleotides were removed using micro-spin G50 columns (Amersham Biosciences, Chalfont St Giles, UK). Labeled DNAs were combined, mixed with $135 \mu \mathrm{g}$ of human Cot-1 DNA (Invitrogen, Paisley, UK), precipitated using ethanol and ressuspended in hybridization buffer containing $50 \%$ formamide, $10 \%$ dextran sulphate, $2 \times$ SSC, $4 \%$ SDS, and $10 \mu \mathrm{g} / \mu \mathrm{L}$ yeast tRNA (Invitrogen, Paisley, UK). Samples were denatured at $72^{\circ} \mathrm{C}$ for 10 minutes and incubated at $37^{\circ} \mathrm{C}$ for 60 minutes before being hybridized onto the slides in a GeneTAC Hybridization station (Genomic Solutions Ltd, Huntingdon, UK). Hybridization took place over 36 hours, followed by auto- 
mated post-hybridization washes in $50 \%$ formamide $/ 2 \times$ SSC $\left(45^{\circ} \mathrm{C}\right), 2 \times \operatorname{SSC} / 0.1 \% \operatorname{SDS}\left(37^{\circ} \mathrm{C}\right)$, and PN buffer $\left(37^{\circ} \mathrm{C}\right)$. Slides were dried by centrifugation after a brief wash in $0.05 \times$ SSC and scanned with an Agilent G2565BA microarray scanner (Agilent Technologies, Palo Alto, CA). Five control hybridizations (normal male versus normal female DNA) were performed, as well as five dye-swap experiments using randomly selected samples. Data from 11 additional negative controls run during the same period with different batches of reference DNA were kindly provided by the Microarray Core Facility to validate the clone set.

\section{Image analysis and processing}

Analysis of the microarray images was performed in GenePix Pro 6.0 (Axon Instruments Inc., Foster City, CA), with the median pixel intensities for each channel (with background subtraction) being calculated for each spot. For each sample, Genepix results were exported as a TABdelimited "GPR" file into Normalisation Suite [41], where background-subtracted channel intensities were normalized (local linear normalization) and combined to produce the final intensity ratios for each feature. For the automated scoring of copy number aberrations, three methods were compared: sample-specific fixed-thresholds, calculated as 2.5 times the baseline noise levels for each sample (Normalisation Suite [42]); a data segmentation approach using K-means clustering (CGH-Plotter [41]), and breakpoint estimation (aCGH-Smooth [43]). The final choice for automated scoring fell upon aCGHSmooth. Graphical visualization of the $\log -2$ ratios for each sample and the overall results for all samples (clones indexed by their physical location along the genome) were generated in Normalisation Suite and Microsoft Excel, respectively. Amplifications were scored whenever $\log -2$ intensity ratio was larger than 0.75 . For determination of homozygous deletions, the average log-2 intensity ratios for deleted regions was calculated for each sample, and clones reaching at least twice this value were scored.

\section{Fluorescent in situ hybridization}

For ten selected biopsy samples, four-micron thick sections from a representative paraffin-embedded block were cut onto SuperFrost Plus Adhesion slides (Menzel-Glaser, Braunschweig, Germany). Sample processing, hybridization, and analysis were performed as described previously [6]. A locus-specific probe for the TP53 gene (17p13.1) and a control probe for the centromere of chromosome 17 (Vysis, Downers Grove, IL) were applied onto each sample, and fluorescent images corresponding to DAPI, SpectrumGreen (CEP17), and SpectrumOrange (17p13.1) were sequentially captured using the same equipment described for CCGH analysis. Only intact, nonoverlapping nuclei were scored. An abnormal population was considered representative when at least three nuclei within the same microscope field presented a given aberration and at least 40 nuclei presented that particular alteration in the whole sample.

\section{TP53 mutation status}

From the 51 samples subject to mutation analysis, direct sequencing (sense and anti-sense) was performed for each of exons 5-8 in 14 samples. The remaining 37 samples were screened for aberrant bands using the temporal temperature gradient electrophoresis (TTGE) method for exons 5,6 , and 8 , whereas exon 7 was directly sequenced. The TTGE method has a better resolution level than sequencing, and aberrant bands may be detected in a sample with $<5 \%$ mutated alleles [44].

\section{Competing interests}

The author(s) declare that they have no competing interests.

\section{Authors' contributions}

FRR carried out the microarray experiments, performed data analysis, and drafted the manuscript. RH collected and graded all prostate carcinomas, gathered clinical information for these patients, and assisted in drafting the manuscript. CJ assisted with sample DNA extraction and drafting the manuscript. $\mathrm{MB}$ assisted with the microarray experiments and data analysis. MH performed the mutation screening of the TP53 gene. MRT and RAL participated in the design and coordination of the study, assisted with analysis, and contributed to manuscript writing. All authors read and approved the final manuscript.

\section{Additional material}

\section{Additional File 1}

Comparison of cCGH and aCGH findings in 46 prostate cancer samples. Detailed description of $c \mathrm{CGH}$ and aCGH findings for each individual sample analysed in this study.

Click here for file

[http://www.biomedcentral.com/content/supplementary/14764598-5-33-S1.pdf]

\section{Acknowledgements}

The valuable technical assistance of Stine Kresse and Leonardo MezaZepeda, from the Department of Tumor Biology of the Institute for Cancer Research, The Norwegian Radium Hospital, is gratefully acknowledged. This work was supported by research grants POCTI/CBO/38853/200I and POCTI/SAU-OBS/58357/2004 awarded by Fundação para a Ciência e a Tecnologia (FCT) and by a grant from the Norwegian Cancer Society (RAL: A95068). FRR (grant SFRH/BD7067/200I) is a research fellow of FCT.

\section{References}

I. Kallioniemi A, Kallioniemi OP, Sudar D, Rutovitz D, Gray JW, Waldman F, Pinkel D: Comparative genomic hybridization for 
molecular cytogenetic analysis of solid tumors. Science 1992, 258:8|8-82I.

2. Ribeiro FR, Diep CB, Jeronimo C, Henrique R, Lopes C, Eknaes M, Lingjaerde OC, Lothe RA, Teixeira MR: Statistical dissection of genetic pathways involved in prostate carcinogenesis. Genes Chromosomes Cancer 2006, 45: I 54-I63.

3. Alers JC, Rochat J, Krijtenburg PJ, Hop WC, Kranse R, Rosenberg C, Tanke HJ, Schroder FH, Van Dekken H: Identification of genetic markers for prostatic cancer progression. Lab Invest 2000, 80:931-942

4. Dong JT, Chen C, Stultz BG, Isaacs JT, Frierson HF Jr: Deletion at |3q2| is associated with aggressive prostate cancers. Cancer Res 2000, 60:3880-3883.

5. Van Dekken H, Alers JC, Damen IA, Vissers KJ, Krijtenburg PJ, Hoedemaeker RF, Wildhagen MF, Hop WC, van der Kwast TH, Tanke HJ, Schroder FH: Genetic evaluation of localized prostate cancer in a cohort of forty patients: gain of distal $8 \mathbf{q}$ discriminates between progressors and nonprogressors. Lab Invest 2003, 83:789-796.

6. Ribeiro FR, Jeronimo C, Henrique R, Fonseca D, Oliveira J, Lothe RA, Teixeira MR: $\mathbf{8 q}$ gain is an independent predictor of poor survival in diagnostic needle biopsies from prostate cancer suspects. Clin Cancer Res 2006, I2:396I-3970.

7. Brookman-Amissah N, Duchesnes $C$, Williamson MP, Wang $Q$, Ahmed A, Feneley MR, Mackay A, Freeman A, Fenwick K, Iravani M, Weber B, Ashworth A, Masters JR: Genome-wide screening for genetic changes in a matched pair of benign and prostate cancer cell lines using array CGH. Prostate Cancer Prostatic Dis 2005, 8:335-343.

8. Zhao H, Kim Y, Wang P, Lapointe J, Tibshirani R, Pollack JR, Brooks JD: Genome-wide characterization of gene expression variations and DNA copy number changes in prostate cancer cell lines. Prostate 2004, 63:187-197.

9. Clark J, Edwards S, Feber A, Flohr P, John M, Giddings I, Crossland S, Stratton MR, Wooster R, Campbell C, Cooper CS: Genome-wide screening for complete genetic loss in prostate cancer by comparative hybridization onto cDNA microarrays. Oncogene 2003, 22: 1247-1252.

10. Wolf M, Mousses S, Hautaniemi S, Karhu R, Huusko P, Allinen M, Elkahloun A, Monni O, Chen Y, Kallioniemi A, Kallioniemi OP: Highresolution analysis of gene copy number alterations in human prostate cancer using CGH on cDNA microarrays: impact of copy number on gene expression. Neoplasia 2004, 6:240-247.

II. Saramaki OR, Porkka KP, Vessella RL, Visakorpi T: Genetic aberrations in prostate cancer by microarray analysis. Int J Cancer 2006, I 19:1322-1329.

12. Yano S, Matsuyama H, Matsuda K, Matsumoto H, Yoshihiro S, Naito $\mathrm{K}$ : Accuracy of an array comparative genomic hybridization (CGH) technique in detecting DNA copy number aberrations: comparison with conventional CGH and loss of heterozygosity analysis in prostate cancer. Cancer Genet Cytogenet 2004, 150:122-127.

13. Van Dekken H, Paris PL, Albertson DG, Alers JC, Andaya A, Kowbel $D$, van der Kwast TH, Pinkel D, Schroder FH, Vissers KJ, Wildhagen MF, Collins C: Evaluation of genetic patterns in different tumor areas of intermediate-grade prostatic adenocarcinomas by high-resolution genomic array analysis. Genes Chromosomes Cancer 2004, 39:249-256.

14. Watson JE, Doggett NA, Albertson DG, Andaya A, Chinnaiyan A, Van Dekken H, Ginzinger D, Haqq C, James K, Kamkar S, Kowbel D, Pinkel D, Schmitt L, Simko JP, Volik S, Weinberg VK, Paris PL, Collins C: Integration of high-resolution array comparative genomic hybridization analysis of chromosome $16 \mathrm{q}$ with expression array data refines common regions of loss at 16 q23-qter and identifies underlying candidate tumor suppressor genes in prostate cancer. Oncogene 2004, 23:3487-3494.

15. van Duin M, van Marion R, Vissers K, Watson JE, van Weerden WM, Schroder FH, Hop WC, van der Kwast TH, Collins C, Van Dekken H High-resolution array comparative genomic hybridization of chromosome arm 8q: Evaluation of genetic progression markers for prostate cancer. Genes Chromosomes Cancer 2005, 44:438-449.

16. Paris PL, Andaya A, Fridlyand J, Jain AN, Weinberg V, Kowbel D, Brebner JH, Simko J, Watson JE, Volik S, Albertson DG, Pinkel D, Alers JC, van der Kwast TH, Vissers KJ, Schroder FH, Wildhagen MF,
Febbo PG, Chinnaiyan AM, Pienta KJ, Carroll PR, Rubin MA, Collins $\mathrm{C}$, Van Dekken $\mathrm{H}$ : Whole genome scanning identifies genotypes associated with recurrence and metastasis in prostate tumors. Hum Mol Genet 2004, 13:1303-1313.

17. Paris PL, Albertson DG, Alers JC, Andaya A, Carroll P, Fridlyand J, Jain AN, Kamkar S, Kowbel D, Krijtenburg PJ, Pinkel D, Schroder FH, Vissers KJ, Watson VJ, Wildhagen MF, Collins C, Van Dekken H: High-resolution analysis of paraffin-embedded and formalinfixed prostate tumors using comparative genomic hybridization to genomic microarrays. Am J Pathol 2003, 162:763-770.

18. Lai WR, Johnson MD, Kucherlapati R, Park PJ: Comparative analysis of algorithms for identifying amplifications and deletions in array CGH data. Bioinformatics 2005, $21: 3763-3770$.

19. Steiner T, Junker K, Burkhardt F, Braunsdorf A, Janitzky V, Schubert J: Gain in chromosome 8q correlates with early progression in hormonal treated prostate cancer. Eur Urol 2002, 41:|67-|7|.

20. Quinn DI, Henshall SM, Sutherland RL: Molecular markers of prostate cancer outcome. Eur J Cancer 2005, 41:858-887.

21. Dong JT: Prevalent mutations in prostate cancer. / Cell Biochem 2006, 97:433-447.

22. Chaib H, MacDonald JW, Vessella RL, Washburn JG, Quinn JE, Odman A, Rubin MA, Macoska JA: Haploinsufficiency and reduced expression of genes localized to the $8 \mathrm{p}$ chromosomal region in human prostate tumors. Genes Chromosomes Cancer 2003, 37:306-313.

23. Magee JA, Abdulkadir SA, Milbrandt J: Haploinsufficiency at the Nkx3.I locus. A paradigm for stochastic, dosage-sensitive gene regulation during tumor initiation. Cancer Cell 2003 , 3:273-283.

24. Li J, Yen C, Liaw D, Podsypanina K, Bose S, Wang SI, Puc J, Miliaresis C, Rodgers L, McCombie R, Bigner SH, Giovanella BC, Ittmann M, Tycko B, Hibshoosh H, Wigler MH, Parsons R: PTEN, a putative protein tyrosine phosphatase gene mutated in human brain, breast, and prostate cancer [see comments]. Science 1997, 275: 1943-1947.

25. Steck PA, Pershouse MA, Jasser SA, Yung WK, Lin H, Ligon AH, Langford LA, Baumgard ML, Hattier T, Davis T, Frye C, Hu R, Swedlund, Teng DH, Tavtigian SV: Identification of a candidate tumour suppressor gene, MMACI, at chromosome I0q23.3 that is mutated in multiple advanced cancers. Nat Genet 1997, I5:356-362.

26. Dreher T, Zentgraf H, Abel U, Kappeler A, Michel MS, Bleyl U, Grobholz R: Reduction of PTEN and p27kip I expression correlates with tumor grade in prostate cancer. Analysis in radical prostatectomy specimens and needle biopsies. Virchows Arch 2004, 444:509-517.

27. Halvorsen OJ, Haukaas SA, Akslen LA: Combined loss of PTEN and p27 expression is associated with tumor cell proliferation by $\mathrm{Ki}-67$ and increased risk of recurrent disease in localized prostate cancer. Clin Cancer Res 2003, 9:1474-|479.

28. Kwabi-Addo B, Giri D, Schmidt K, Podsypanina K, Parsons R, Greenberg N, Ittmann M: Haploinsufficiency of the Pten tumor suppressor gene promotes prostate cancer progression. Proc Natl Acad Sci USA 200 I, 98: I I563-I I 568.

29. Kim MJ, Cardiff RD, Desai N, Banach-Petrosky WA, Parsons R, Shen MM, Abate-Shen C: Cooperativity of Nkx3.I and Pten loss of function in a mouse model of prostate carcinogenesis. Proc Natl Acad Sci USA 2002, 99:2884-2889.

30. Hill R, Song Y, Cardiff RD, Van Dyke T: Heterogeneous tumor evolution initiated by loss of pRb function in a preclinical prostate cancer model. Cancer Res 2005, 65: 10243-10254.

31. Cairns P, Okami K, Halachmi S, Halachmi N, Esteller M, Herman JG, Jen J, Isaacs WB, Bova GS, Sidransky D: Frequent inactivation of PTEN/MMACI in primary prostate cancer. Cancer Res 1997 , 57:4997-5000.

32. Dong JT, Sipe TW, Hyytinen ER, Li CL, Heise C, McClintock DE, Grant CD, Chung LW, Frierson HFJ: PTEN/MMACI is infrequently mutated in pT2 and pT3 carcinomas of the prostate. Oncogene 1998, 17:1979-1982.

33. Feilotter HE, Nagai MA, Boag AH, Eng C, Mulligan LM: Analysis of PTEN and the $10 \mathrm{q} 23$ region in primary prostate carcinomas. Oncogene 1998, 16:1743-1748.

34. Gray IC, Stewart LM, Phillips SM, Hamilton JA, Gray NE, Watson GJ, Spurr NK, Snary D: Mutation and expression analysis of the 
putative prostate tumour- suppressor gene PTEN. $\mathrm{Br} J$ Cancer 1998, 78: 1296-1300.

35. Vlietstra RJ, van AD, Hermans KG, van SG, Trapman J: Frequent inactivation of PTEN in prostate cancer cell lines and xenografts. Cancer Res 1998, 58:2720-2723.

36. Hermans KG, van Alewijk DC, Veltman JA, van Weerden W, van Kessel AG, Trapman J: Loss of a small region around the PTEN locus is a major chromosome 10 alteration in prostate cancer xenografts and cell lines. Genes Chromosomes Cancer 2004, 39:17I-184.

37. Verhagen $P$, van Duijn P, Hermans K, Looijenga L, van Gurp R, Stoop $\mathrm{H}$, van der KT, Trapman J: The PTEN gene in locally progressive prostate cancer is preferentially inactivated by bi-allelic gene deletion. J Pathol 2006, 208:699-707.

38. Norwegian Microarray Consortium [http://www.microar ray.no]

39. Fiegler H, Carr P, Douglas EJ, Burford DC, Hunt S, Scott CE, Smith J, Vetrie D, Gorman P, Tomlinson IP, Carter NP: DNA microarrays for comparative genomic hybridization based on DOP-PCR amplification of BAC and PAC clones. Genes Chromosomes Cancer 2003, 36:36I-374.

40. Ensembl Human Genome Browser [http://www.ensembl.org]

41. Beheshti B, Braude I, Marrano P, Thorner P, Zielenska M, Squire JA: Chromosomal localization of DNA amplifications in neuroblastoma tumors using cDNA microarray comparative genomic hybridization. Neoplasia 2003, 5:53-62.

42. Autio R, Hautaniemi S, Kauraniemi P, Yli-Harja O, Astola J, Wolf M, Kallioniemi A: CGH-Plotter: MATLAB toolbox for CGH-data analysis. Bioinformatics 2003, 19:17/4-17/5.

43. Jong K, Marchiori E, Meijer G, Vaart AV, Ylstra B: Breakpoint identification and smoothing of array comparative genomic hybridization data. Bioinformatics 2004, 20:3636-3637.

44. Sørlie T, Johnsen H, Vu P, Lind GE, Lothe RA, Børresen-Dale A-L: Mutation screening of the TP53 gene by Temporal Temperature Gel Electrophoresis (TTGE). In Methods in Molecular Biology Volume 29I. Humana Press, Totowa, NJ; 2005:207-216.

Publish with Bio Med Central and every scientist can read your work free of charge

"BioMed Central will be the most significant development for disseminating the results of biomedical research in our lifetime. "

Sir Paul Nurse, Cancer Research UK

Your research papers will be:

- available free of charge to the entire biomedical community

- peer reviewed and published immediately upon acceptance

- cited in PubMed and archived on PubMed Central

- yours - you keep the copyright

Submit your manuscript here:

http://www.biomedcentral.com/info/publishing_adv.asp
BioMedcentral 